\title{
Den Zusammenhang verstehen, die Fachkenntnis vertiefen und eine positive Kommunikation im Team fördern
}

\section{Blickfang Pathogenese FOKUS}

\section{Multiple Sklerose}

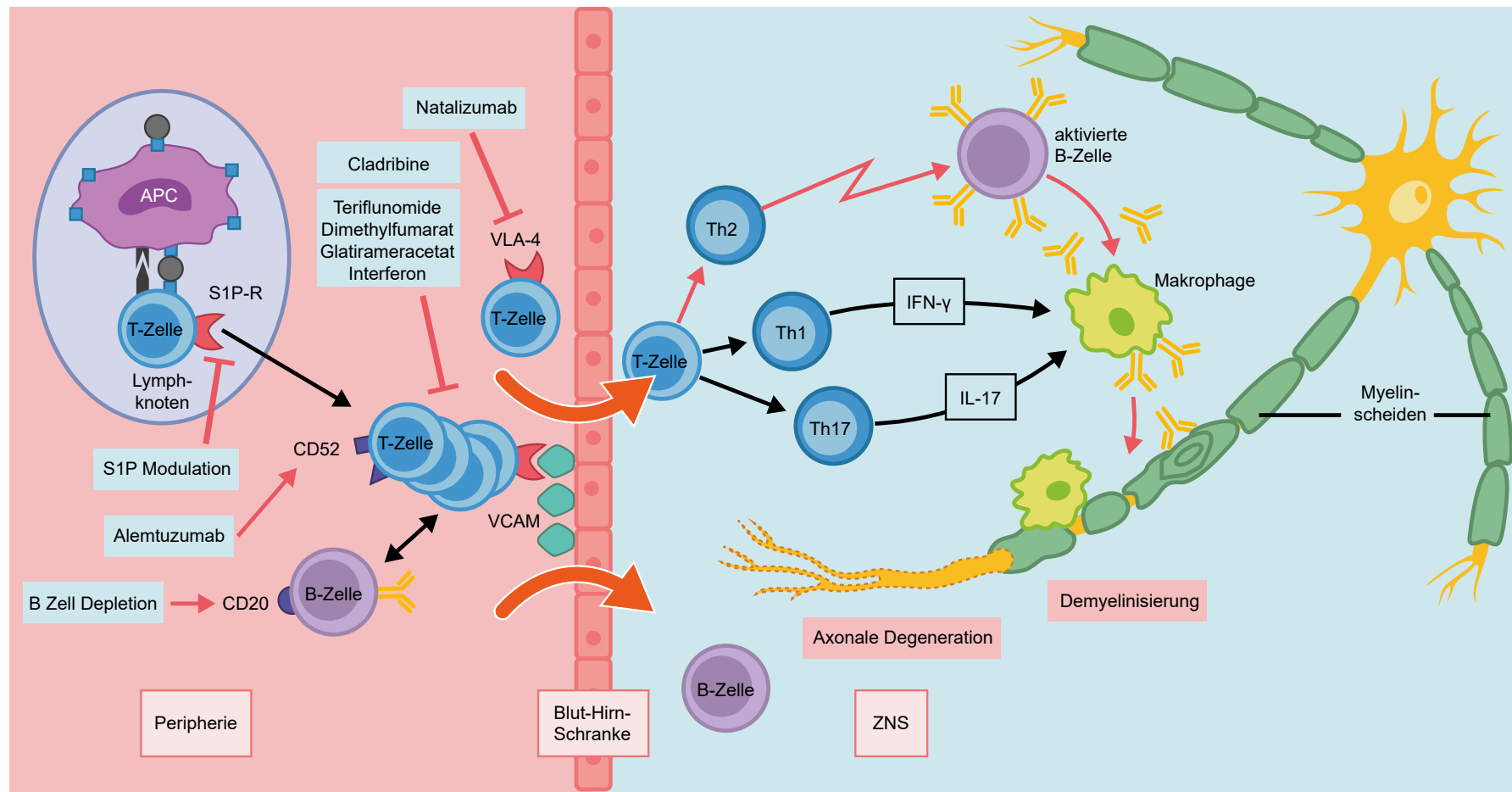

Grafik modifiziert nach

Linker RA, Kieseier BC, Gold R: Identification and development of new therapeutics for multiple sclerosis. Trends Pharmacol Sci. 2008;29(11):558-565.

Bendas G: Multiple Sklerose - Therapie, die an die Nerven geht. Pharmazeutische Zeitung 03/2018 online.

Die Multiple Sklerose (MS) ist charakterisiert durch einen wiederholt an unterschiedlichen Orten im zentralen Nervensystem auftretenden chronischen Entzündungsprozess, der zur Zerstörung der Markscheiden und im weiteren Verlauf auch der Axone führt. Dadurchentstehen diefürdieMStypischengroßenDemyelinisierungsherde im Rückenmark und Gehirn, wobei, so der derzeitige Wissensstand, eine autoimmune Pathogenese - moduliert durch genetische Disposition und Umweltfaktoren - eine Rolle spielt. Dabei können autoreaktive T-Lymphozyten, die auch beim gesunden Menschen Bestandteil des Immunsystems sind, nach der Aktivierung in der Peripherie die Blut-Hirn-Schranke überwinden und somit ins zentrale Nervensystem eindringen. Wenn diese peripher aktivierten T-Lymphozyten ihr spezifisches Antigen vorfinden, be- ginnt die entzündliche Reaktion, bei der auch B-Zellen und Makrophagen eine wichtige Rolle spielen. Die aktuellen Immuntherapeutika wirken v.a. in der Peripherie, zentrale Wirkmechanismen werden für einige Therapeutika angenommen, sind aber nicht bewiesen. Neben der klassischen Immunmodulation kommen depletierende Therapien zum Einsatz wie Cladribine, Alemtuzumab und Ocrelizumab, die unterschiedliche Immunpopulationen depletieren. Die Migration von Immunzellen in das zentrale Nervensystem wird durch Natalizumab und die S1P-Modulatoren wie z.B. Fingolimod, Siponimod und Ozanimod inhibiert.

Autor: Prof. Dr. Tjalf Ziemssen, Zentrum für klinische Neurowissenschaften, Klinik und Poliklinik für Neurologie, Universitätsklinikum Dresden 


\section{Fort- und Weiterbildung FOKUS}

\section{Weiterbildung zum MS-Spezialisten mit großem Praxisbezug}

Bei Multipler Sklerose (MS) hemmt eine früh im Krankheitsverlauf begonnene Therapie das Voranschreiten der Erkrankung. Daher sind eine zeitnahe Diagnose und Behandlung extrem wichtig für den Patienten. Die Ausbildung von MS-Spezialisten stellt einen wichtigen Baustein im Kampf gegen die autoimmunbedingte Erkrankung dar. Seit Oktober bietet die Dresden International University (DIU), die Weiterbildungsuniversität der TU Dresden, erstmals den Masterstudiengang «Multiple Sklerose Management» an. Der berufsbegleitende Studiengang richtet sich an deutschsprachige Ärzte, Therapeuten, Pflegepersonal und Wissenschaftler in Klinik, Wissenschaft und Praxis, in der Pharmaindustrie und öffentlicher Anstellung sowie Apotheker, Psychologen und Biologen, die sich auf Multiple Sklerose spezialisieren wollen.

«Wir haben zum Studienstart 15 Anmeldungen, die aus allen genannten Bereichen stammen», berichtet Franziska Ramisch, Weiterbildungsmanagerin an der DIU. Zulassungskriterien für die Teilnehmenden sind ein humanmedizinisches Studium oder ein akademischer Abschluss plus ein Abschluss in einem Gesundheitsfachberuf. «Voraussetzung für die Teilnahme ist zudem 1 Jahr Berufserfahrung im Umgang mit MS», ergänzt Ramisch. Der Studiengang wird über 4 Semester berufsbegleitend durchgeführt, d.h. der Unterricht findet vorrangig an 3 bis 4 Wochenenden im Semester sowie in Abendveranstaltungen an Wochentagen statt. Die Lehrveranstaltungen werden größtenteils online-basiert und in der Regel in deutscher Sprache durchgeführt. «Wenn sich die Weiterbildung etabliert hat, planen wir auch eine Internationalisierung», berichtet Ramisch.

Der Studiengang vermittelt die Kenntnis der wissenschaftlich fundierten Diagnostik und Differentialdiagnostik bei Verdacht auf chronisch-entzündliche Erkrankungen des zentralen Nervensystems (ZNS) mit Schwerpunkt MS. Die Absolventen erlernen das «State of

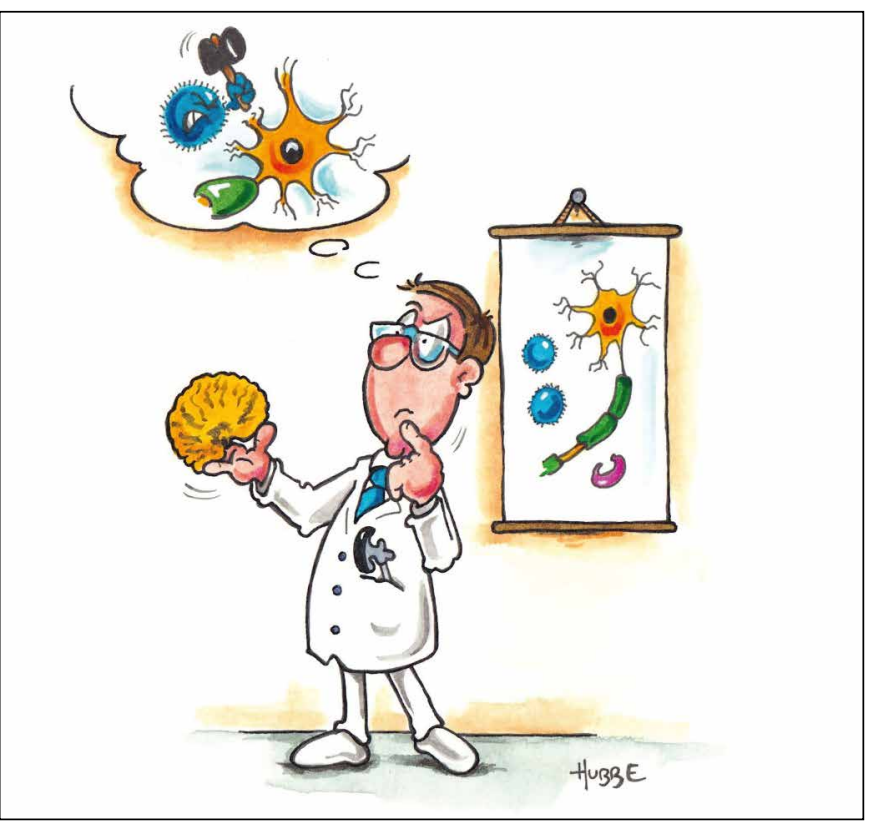

Nach absolvierter Weiterbildung weiß der MS-Manager, wie er seine Patienten am effektivsten begleitet. @ Philipp Hubbe/www.hubbe-cartoons.de the art Management» chronisch-entzündlicher ZNS-Erkrankungen einschließlich der Therapiemaßnahmen und -strategien für den individuellen Patienten. Ebenso gehört das engmaschigen Monitoring der Krankheitsaktivität und -behinderung zu den Lerninhalten. Ein weiteres Ziel ist die Befähigung, wissenschaftliche Publikationen und klinischen Studien zu beurteilen und sie im Kontext des individuellen Krankheitsgeschehens zu interpretieren. Darauf aufbauend erarbeiten die Studierenden therapeutische Interventionspläne und wenden sie in der direkten Patientenversorgung an.

Der Studiengang gliedert sich in 6 Module mit theoretischem Unterricht und zahlreichen Praxiseinheiten sowie eine abschließende Masterarbeit. Alle Module werden entweder mit einer Klausur, einer mündlichen Prüfung oder einem Fallbericht abgeschlossen.

\section{Die Schwerpunkte der Module}

- Modul I: MS Grundlagen

- Modul II: MS Klinik und Diagnostik

- Modul III: MS Studien und Statistik

- Modul IV: MS Therapie I

- Modul V: MS Therapie II

- Modul VI: MS Monitoring und Dokumentation

Neben der klassischen Wissensvermittlung durch erfahrene MSExperten in Vorlesungen und Tutorien dienen Hospitationen in speziell ausgewählten Zentren mit großer Erfahrung in Diagnostik und Therapie der direkten praktischen Umsetzung der erlernten Inhalte vor Ort. «Professor Ziemssen, wissenschaftlicher Leiter des Studiengangs, konnte viele namhafte Experten aus seinem Netzwerk als Referenten gewinnen», berichtet Ramisch. Regelmäßig sind Exkursionen zu Kongressen (z.B. ECTRIMS - European Commitee for Treatment and Research in Multiple Sclerosis) und Expertengespräche mit beruflich Erfahrenen geplant, wodurch zum einen das Erlernte nochmals reflektiert und vertieft werden kann und zum anderen die Einbindung von Innovationen, z.B. im Bereich Therapie und Medikation, garantiert ist. Der starke Praxisbezug des Studiengangs spiegelt sich außerdem in der Durchführung digitaler Fallkonferenzen und Journalclubs wider. Durch die Lehr- und Lernunterlagen wird ein effektives Selbststudium möglich, das der Wissensverbreiterung und -vertiefung dient.

Die Schirmherrschaft über den Studiengang übernimmt die Deutsche Multiple Sklerose Gesellschaft (DMSG). Unterstützt wird der Studiengang vom Universitätsklinikum Carl Gustav Carus Dresden, von der Deutschen Gesellschaft für Neurologie (DGN), dem Berufsverband Deutscher Nervenärzte (BVDN), dem Berufsverband Deutscher Neurologen (BDN), von ParadigMS und der European Multiple Sclerosis Platform (EMSP).

Autorin: Christine Schiller, S.Karger Verlag Freiburg

Weitere Informationen:www.di-uni.de/studium-weiterbildung/medizin/ ms-management 


\section{Aus der Praxis}

\section{Der Umgang mit dem Pflegepersonal als Arzt}

Beginnt man seine Assistenz in einem Klinikum, trifft man oft auf sehr erfahrene Krankenschwestern und Krankenpfleger, die einem medizinische Tipps geben. Sollte man sie annehmen?

Um es vorwegzunehmen: Man kann die eingangs gestellte Frage nicht mit einem klaren «Ja» oder «Nein» beantworten, weil auch hier der Ton die Musik macht. Wenn eine erfahrene Schwester Vorschläge macht, was die Diagnose und Therapie von Patienten angeht, dann sollte man sich diese Vorschläge anhören. Pflegekräfte haben oft eine lange Berufserfahrung und die zunehmende Akademisierung ihrer Ausbildung macht den immer breiteren Erwerb von Fachwissen möglich. Dennoch bist du der Arzt oder die Ärztin und hast damit die Durchführungsverantwortung, während das Pflegepersonal in der Anordnungsverantwortung ist, d.h. du haftest später für die getroffene Entscheidung und nicht das Pflegepersonal. Wichtig ist deshalb, dass man Ratschläge anderer nicht vorschnell annimmt, sondern sich selbst in aller Ruhe ein Bild macht. Im Zweifel fragt man den Oberarzt oder den Weiterbildungsbevollmächtigten, ob die angedachte Therapie sinnvoll ist.

\section{Parallele Hierarchien}

Bei dem Pflegepersonal gibt es eine Pyramidenhierachie, die von der Hilfsschwester über die Schwester, zur Stationsschwester, zur Pflegedienstleitung bis zur Pflegedirektion reicht. Wie bei den Ärzten gibt es auch hier eine Weisungsbefugnis von oben nach unten. Grundsätzlich gilt: Die pflegerische Versorgung übernimmt das Pflegepersonal selbstständig, die medizinische Aufgaben werden hingegen von dir als Arzt oder Ärztin delegiert, und zwar sowohl an die Hilfsschwester als auch an die Stationsschwester.

\section{Konflikte mit dem Pflegepersonal: 2 Beispiele}

Es ist oft gar nicht so leicht, als «Frischling» einer Stationsschwester Aufgaben zu erteilen. Deshalb ist mit großem Fingerspitzengefühl vorzugehen. Aber manchmal kann auch das größte diplomatische Geschick keine direkte Auseinandersetzung verhindern. Zur Verdeutlichung ein Beispiel: Nehmen wir an, du beginnst deine Assistenz und die Stationsschwester, die erheblich älter ist, will von dir gesiezt werden, während sie dich mit «Du» anspricht. Auf sehr direkte Weise versucht sie also das Machtverhältnis umzukehren. Hier empfiehlt sich der direkte Weg der Kommunikation. Mach ihr deutlich, dass ihr euch in einem Dienstverhältnis befindet und du deshalb das höflichere «Sie» bevorzugst. Du wirst sie mit diesem Vorgehen nicht als Freundin gewinnen, aber sie hat es ja selbst auf die Konfrontation angelegt und ist damit eine schwierige Kollegin, mit der du ohnehin nicht warm geworden wärst.

Andererseits kann die Expertise des Fachpflegepersonals lebensentscheidend sein. In tragischer Weise verdeutlicht dies der Tod der Britin Elaine Bromiley. Sie verstarb aufgrund von Fehlern bei der Narkoseeinleitung, die teilweise vermeidbar gewesen wären, wenn die behandelnden Anästhesisten auf die Ratschläge der Anästhesieschwestern gehört hätten. Letztendlich geht es auf Station um Teamwork, bei der jeder sein Wissen einbringen dürfen sollte. Eine junge Ärztegeneration, die um ihre Verantwortung weiß, klare Grenzen zieht, aber auch gegenüber Kritik aufgeschlossen ist, kann in Zukunft sicherlich viel bewirken.

Originalquelle: Der Umgang mit dem Pflegepersonal als Arzt. Ein Beitrag auf www.operation-karriere.de (www.operation-karriere.de/ karriereweg/bewerbung-berufsstart/der-umgang-mit-dem-pflegepersonal-als-arzt.html)

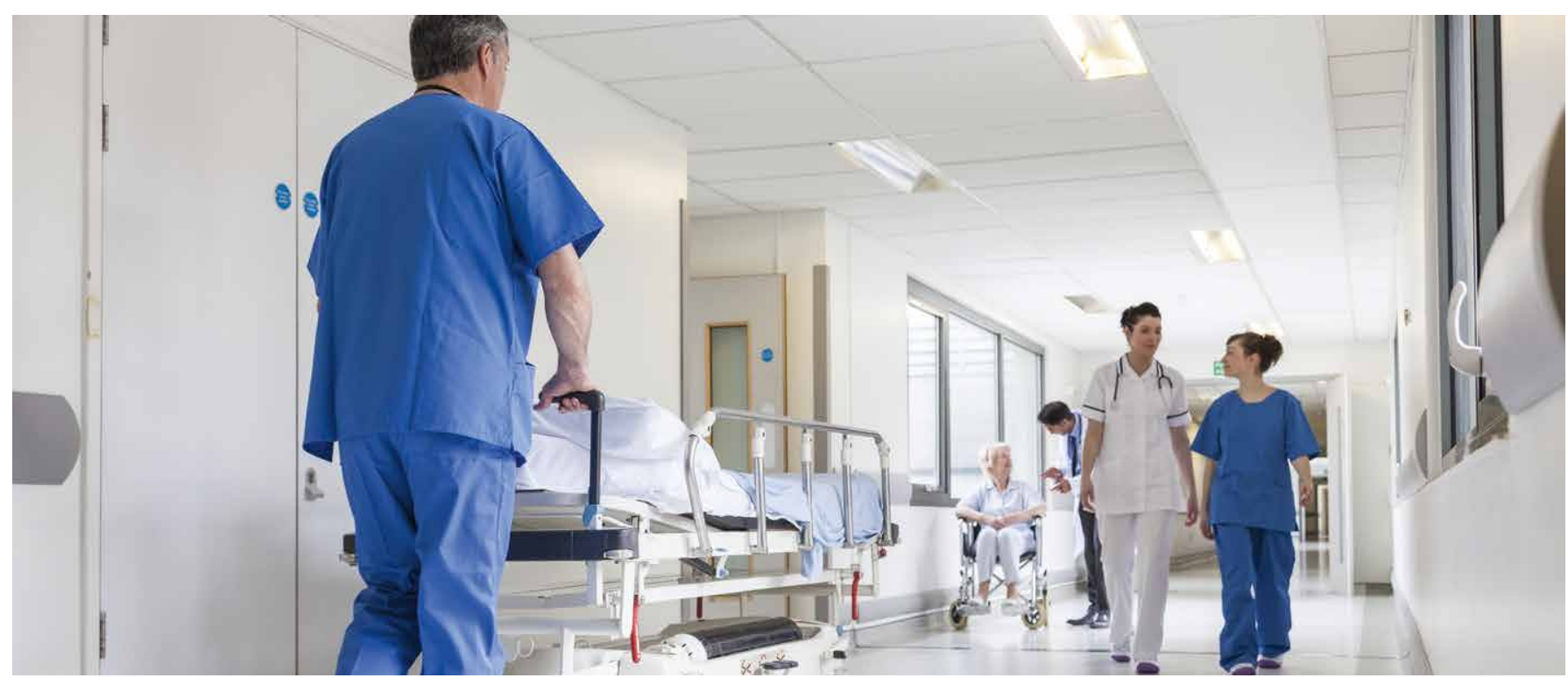

Duzt oder siezt man sich? Der Umgangston ist von Station zu Station unterschiedlich. @ Spotmatik/Shutterstock 